\title{
Serum interleukin 10 and interleukin 11 in patients with acute pancreatitis
}

\author{
C-C Chen, S-S Wang, R-H Lu, F-Y Chang, S-D Lee
}

\begin{abstract}
Background-Proinflammatory and antiinflammatory cytokines are involved in the pathogenesis of acute pancreatitis. Aims-To measure the serial serum levels of interleukin 10 and interleukin 11 in patients with acute pancreatitis and analyse the relation of these antiinflammatory cytokines to disease severity.

Methods-In 50 patients with acute pancreatitis, the serum concentrations of interleukin 10 and interleukin 11 were determined on days one, two, three, four, and seven after admission. Serum $C$ reactive protein levels were evaluated on days one and two. Severity of pancreatitis was determined according to the Atlanta criteria.
\end{abstract}

Results-Serum concentrations of interleukin 10 on days one to seven were significantly higher in patients with severe pancreatitis than in those with mild pancreatitis. Patients with severe attacks had significantly elevated serum interleukin 11 concentrations on days two to four compared with those with mild attacks, but not on days one and seven. With cut off levels of $30 \mathrm{pg} / \mathrm{ml}$ for interleukin $10,10.5 \mathrm{pg} / \mathrm{ml}$ for interleukin 11 , and $115 \mathrm{mg} / \mathrm{l}$ for $\mathrm{C}$ reactive protein, the accuracy rates for detecting severe pancreatitis were $84 \%, 64 \%$, and $78 \%$ respectively on day one and $82 \%, 74 \%$, and $84 \%$ respectively on day two.

Conclusions-Serum interleukin 10 and interleukin 11 concentrations reflect the severity of acute pancreatitis. Interleukin 10 is a useful variable for early prediction of the prognosis of acute pancreatitis.

(Gut 1999;45:895-899)

Keywords: acute pancreatitis; $\mathrm{C}$ reactive protein; interleukin 10 ; interleukin 11

r C-C Chen, Division of Gastroenterology,

Department of Medicine, Veterans General

Hospital-Taipei, No 201, Section 2, Shih-Pai Road, Taipei, 11217, Taiwan.

Accepted for publication 23 June 1999

Table 1 Characteristics of patients with acute pancreatitis

\begin{tabular}{llll}
\hline & Severe $(n=18)$ & Mild $(n=32)$ & Total $(n=50)$ \\
\hline M/F & $15 / 3$ & $25 / 7$ & $40 / 10$ \\
Mean age (years) & 63 & 64 & 64 \\
Aetiology & & & 30 \\
$\quad$ Gallstone & 10 & 20 & 8 \\
Alcohol & 4 & 4 & 9 \\
Idiopathic & 3 & 6 & 3 \\
$\quad$ Miscellaneous & 1 & 2 & \\
\hline
\end{tabular}

completely understood, it is generally accepted that activated leucocytes play an important role in the pathogenesis of acute pancreatitis. ${ }^{12}$ The serum levels of proinflammatory cytokines, including tumour necrosis factor $\alpha$ (TNF- $\alpha$ ), interleukin $1 \beta$ (IL-1 $\beta$ ), interleukin-6 (IL-6), and interleukin-8 (IL-8), have been reported to be significantly higher in severe acute pancreatitis than mild pancreatitis..$^{3-8}$

Interleukin 10 (IL-10) is a recently characterised potent anti-inflammatory cytokine. ${ }^{9}$ In vitro, IL-10 inhibits several functions of macrophages/monocytes, including the production of IL-1, IL-6, IL-8, TNF, colony stimulating factor, intercellular adhesion molecule-1, and nitric oxide. ${ }^{9-11}$ It also decreases the cellular immune response by suppressing IL-2 and interferon- $\gamma$ production. ${ }^{12}$ Plasma IL-10 was shown to be significantly higher in patients with septic shock than in septicaemic patients without shock. ${ }^{13}$ IL-10 has been shown to reduce the severity of experimental acute pancreatitis. ${ }^{14}{ }^{15}$ There are few data in the literature on the changes in serum levels of IL-10 in patients with acute pancreatitis, and the results are conflicting. ${ }^{16} 17$

Interleukin 11 (IL-11) has multiple effects on both haematopoietic and nonhaematopoietic cells. ${ }^{18}$ IL-11 has been found to stimulate the $\mathrm{T}$ cell dependent development of specific immunoglobulin-secreting B cells from murine splenocyte cultures or human peripheral blood cells. ${ }^{19}$ IL-11, like IL-6 and leukaemia inhibitor factor, can stimulate the synthesis of hepatic acute phase protein by hepatoma cells. ${ }^{20}$ Recently, recombinant human IL-11 has been shown to be a potent antiinflammatory cytokine. ${ }^{21}$ In a mouse model of endotoxaemia, pretreatment with recombinant human IL-11 blocked lipopolysaccharide induced elevation of serum levels of TNF- $\alpha$, IL-1 $\beta$, and interferon- $\gamma .{ }^{21}$ To the best of our knowledge, there have been no reports on serum levels of IL-11 in patients with acute pancreatitis in the literature.

The aim of this study was to measure serial serum levels of IL-10 and IL-11 in patients with acute pancreatitis within one week of admission. We also compared the prognostic value of serum $\mathrm{C}$ reactive protein (CRP) with that of the anti-inflammatory cytokines.

Abbreviations used in this paper: APACHE, acute physiology and chronic health evaluation; CRP, C reactive protein; IL, interleukin; TNF, tumour necrosis factor. 


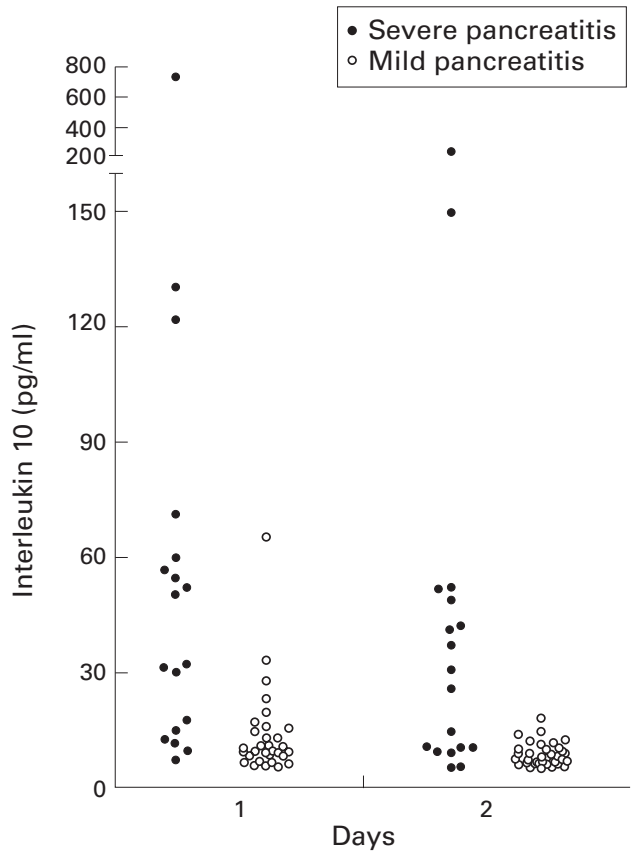

Figure 1 Scatter diagram of serum interleukin $10 \mathrm{in}$ patients with severe or mild pancreatitis on days one and two.

\section{Materials and methods}

Fifty consecutive patients with acute pancreatitis admitted to the Gastroenterology Unit of the Veterans General Hospital-Taipei were recruited. Diagnosis of acute pancreatitis was based on the presence of abdominal pain associated with serum levels of amylase and lipase more than three times the upper normal limit (normal amylase <180 IU/1, lipase <190 IU/1). Abdominal disorders with similar clinical manifestations - for instance, perforated peptic ulcer and intestinal obstruction-were excluded. Only those patients who had suffered an attack of acute pancreatitis within 24 hours

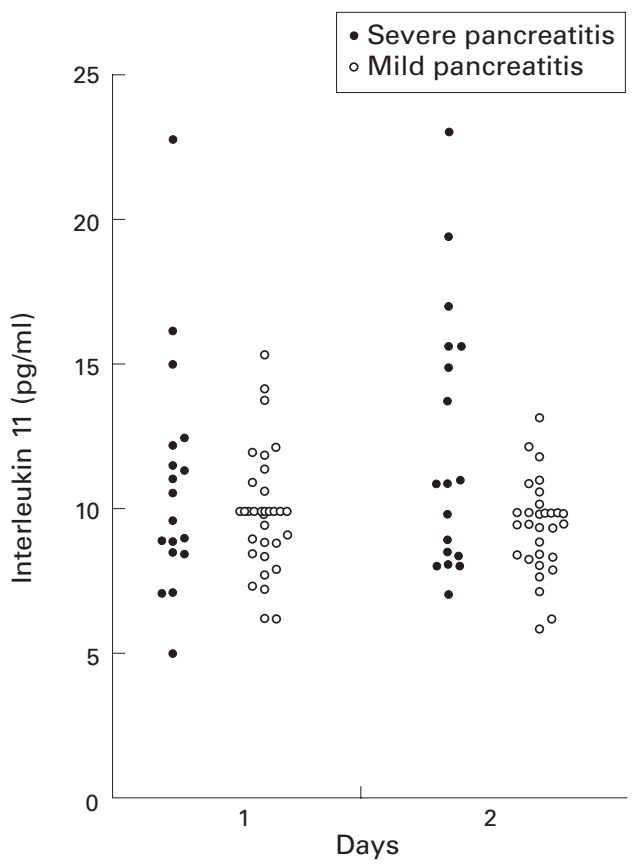

Figure 2 Scatter diagram of serum interleukin 11 in patients with severe or mild pancreatitis on days one and two. were enrolled. Those with pancreatitis caused by trauma, surgery, or endoscopic intubation and those associated with pancreatic tumours, diabetic ketoacidosis, non-ketotic hyperosmolar syndrome, underlying renal function impairment, or pregnancy were excluded. All patients were given intravenous fluids and no oral alimentation. Serum samples for the determination of IL-10 and IL-11 were collected on the day of admission (day one) and on the morning of days two, three, four, and seven of the disease. Sera used for determination of cytokines were stored at $-80^{\circ} \mathrm{C}$ before study. Serum CRP concentrations were measured on days one and two. Appropriate laboratory and physiological data were recorded on days one and two to permit calculation of the acute physiology and chronic health evaluation (APACHE) II score. ${ }^{22}{ }^{23}$ Weightings for age and chronic health state were added to give the final score. Data were collected on admission and 48 hours later to calculate the scores of Ranson criteria. ${ }^{24}$ Abdominal ultrasonography was performed within 72 hours of admission in every case. A contrast enhanced computed tomography scan of the abdomen was performed within one week when the Ranson's score was more than three, when peripancreatic collection was suspected by ultrasonography, or when gas blockade interfered with the examination. Severity of pancreatitis was determined according to the Atlanta criteria. ${ }^{25}$ The criteria of severity include the presence of organ failure and/or the presence of local complications (pancreatic necrosis, abscess, or pseudocyst), and the presence of three or more Ranson criteria or eight or more APACHE II score. The patients were prospectively observed until discharge or death.

Serum levels of IL-10 and IL-11 were determined using commercial solid phase ELISA kits (Quantikine; R\&D Systems, Minneapolis, Minnesota, USA). The minimum detectable values of IL-10 and IL-11 were 1.5 and 8.0 $\mathrm{pg} / \mathrm{ml}$ respectively. Serum CRP was measured with a commercial kit using a Behring Nephelometer-Analyzer (Marburg, Germany). ${ }^{26}$

Data are expressed as mean (SEM). MannWhitney U test was used for statistical analysis. Correlation between serum markers was evaluated by linear regression analysis. $\mathrm{p}<0.05$ was

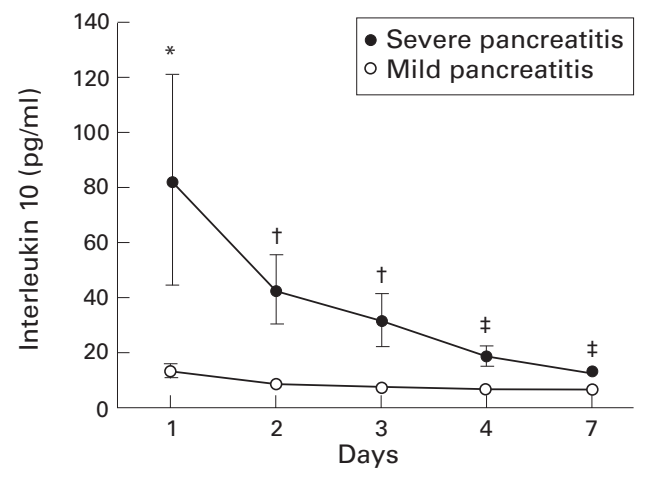

Figure 3 Serial concentrations of serum interleukin 10 in patients with severe or mild pancreatitis within one week of admission. Data are expressed as mean (SEM). ${ }^{\star} p<0.05$, $\neq p<0.005, \neq p<0.0001$ v mild pancreatitis. 


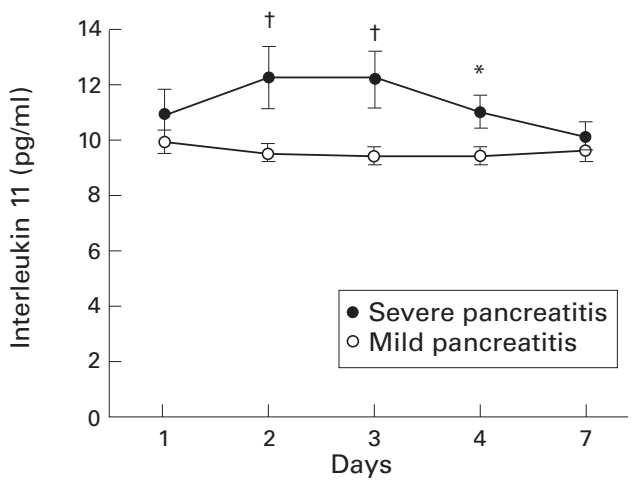

Figure 4 Serial concentrations of serum interleukin 11 in patients with severe or mild pancreatitis within one week of admission. Data are expressed as mean (SEM). $t p<0.005$, ${ }^{\star} p<0.05 v$ mild pancreatitis.

defined as statistically significant. Sensitivity, specificity, positive predictive value, negative predictive value, and accuracy in the determination of severe pancreatitis were defined as described by Ransohoff and Feinstein. ${ }^{27} \mathrm{Re}$ ceiver operating characteristic analysis was used to choose the best cut off values for diagnostic indices. ${ }^{28}$ Sensitivity was defined as the proportion of patients with severe attacks correctly predicted; specificity was the proportion of patients with mild disease correctly predicted; positive predictive value was the proportion of patients with a positive test who indeed had severe disease; negative predictive value was the proportion of patients with a negative test who indeed had mild disease; and accuracy was the proportion of patients correctly classified.

\section{Results}

Forty men and 10 women with a mean age of 63.9 years (range $32-84$ years) were studied. Eighteen patients (36\%) developed severe pancreatitis (including two with septicaemia and three deaths), and 32 patients (64\%) were classified as mild. Table 1 shows the clinical characteristics of these patients. Figures 1 and

Table 2 The acute physiology and chronic health evaluation (APACHE) II scores and serum $C$ reactive protein values on days 1 and 2 in patients with acute pancreatitis

\begin{tabular}{llll}
\hline & Severe $(n=18)$ & Mild $(n=32)$ & $p$ Value \\
\hline APACHE II score & $12.6(1.4)$ & $6.2(0.7)$ & $<0.0005$ \\
$\quad$ Day 1 & $11.3(1.4)$ & $5.4(0.4)$ & $<0.0001$ \\
$\quad$ Day 2 & & $56(10)$ & $<0.0005$ \\
C reactive protein & $125(17)$ & $95(17)$ & $<0.0001$ \\
$\quad$ Day 1 & $242(32)$ & & \\
Day 2 &
\end{tabular}

Table 3 Comparison of serum interleukin 10 (IL-10), 11 (IL-11) and C reactive protein (CRP) for the early prediction of acute pancreatitis

\begin{tabular}{llllll}
\hline & Sensitivity (\%) & Specificity (\%) & PPV (\%) & NPV (\%) & Accuracy (\%) \\
\hline IL-10 & & & & & \\
Day 1 & 67 & 94 & 86 & 83 & 84 \\
Day 2 & 50 & 100 & 100 & 78 & 82 \\
IL-1 1 & & & & & \\
Day 1 & 50 & 72 & 50 & 72 & 64 \\
Day 2 & 56 & 84 & 67 & 71 & 74 \\
CRP & & & 89 & 76 & 78 \\
Day 1 & 44 & 96 & 73 & 93 & 84 \\
Day 2 & 89 & 82 & & \\
\hline
\end{tabular}

The cut off values for IL-10, IL-11 and CRP are $\geqslant 30 \mathrm{pg} / \mathrm{ml}, \geqslant 10.5 \mathrm{pg} / \mathrm{ml}$ and $\geqslant 115 \mathrm{mg} / \mathrm{l}$, respectively.

NPV, negative predictive value; PPV, positive predictive value.
2 are scatter diagrams of serum IL-10 and IL-11 respectively on days one and two in patients with severe and mild acute pancreatitis. Figures 3 and 4 show serum levels of IL-10 and IL-11 respectively on days one, two, three, four, and seven in severe and mild attacks. Serum concentrations of IL-10 on days one, two, three, four, and seven were significantly higher in patients with severe pancreatitis than in those with mild pancreatitis. Patients with severe attacks had significantly elevated serum IL-11 concentrations on days two to four compared with those with mild attacks, but not on days one and seven. The peak serum concentrations (mean values) of IL-10 and IL-11 were 299.8 and $17.1 \mathrm{pg} / \mathrm{ml}$ respectively in patients with a fatal outcome. The APACHE II scores and serum CRP levels on days one and two were significantly higher in patients with severe attacks than in those with mild attacks (table 2).

Table 3 shows the sensitivity, specificity, positive predictive value, negative predictive value, and accuracy of IL-10, IL-11, and CRP in predicting the severity of pancreatitis on days one and two. On day one, the accuracy for serum IL-10 was higher than that for IL-11 and CRP. On day two, the sensitivity and accuracy were higher for serum CRP than for IL-10 and IL-11. Serum IL-10 levels correlated significantly with serum IL-11 levels on day two $(r=0.55$, $\mathrm{p}<0.0001$ ), but not on day one. There was no correlation between serum IL-10 and CRP levels on days one and two. Serum IL-11 concentration showed a weak correlation with serum CRP on days one and two $(r=0.34$, $\mathrm{p}<0.05$ and $r=0.37, \mathrm{p}<0.01$ respectively).

\section{Discussion}

Our results show that serum IL-10 levels were considerably increased in patients with severe acute pancreatitis compared with those with mild attacks. IL-10 peaked on day one and then progressively decreased in the following days in the severe cases. There are few data in the literature about the behaviour of serum IL-10 in patients with acute pancreatitis. Our results differ from those of Pezzilli et al, ${ }^{16}$ who showed that, on the first day of acute pancreatitis, serum levels of IL-10 were significantly higher in patients with mild disease than in those with severe disease, whereas in the following days, no significant difference was observed between the two groups. In contrast, Wereszczynska-Siemiatkowska et $\mathrm{al}^{17}$ showed in a preliminary report that serum IL-10 levels peaked at admission and were higher in severe acute pancreatitis than in mild pancreatitis. The discrepancy in results may be, in part, related to the different criteria used to stage the severity of acute pancreatitis. We prospectively observed the patients until discharge or death, and applied the Atlanta criteria to define the severity of pancreatitis, whereas Pezzilli et $a l^{16}$ used the Balthazar criteria (assessment of computed tomographic examination) to stage the severity. Their definition of the severe form of the disease did not include organ failure or early prognostic signs, such as Ranson score $\geqslant 3$ or APACHE II score $\geqslant 8$. In the early phase after endoscopic retrograde cholangiopancrea- 
tography pancreatitis, peak serum levels of IL-10 correlated significantly with abdominal pain, number of cannulation attempts, and duration of endoscopy. ${ }^{29}$

IL-10 was reported to be significantly higher in patients with septic shock than in septicaemic patients without shock. ${ }^{13}$ IL-10 peaked during the first 48 hours and remained detectable for three to five days after admission. ${ }^{13}$ Similarly, plasma IL-10 concentrations were shown to be related to the severity of septic shock as measured by the APACHE II scoring system. ${ }^{30}$ Systemic inflammatory response syndrome and progression to multiple organ failure syndrome are associated with sepsis, trauma, burns, and severe pancreatitis. ${ }^{31}$ It is conceivable that serum IL-10 level reflects the severity of acute pancreatitis, similar to the pattern observed for the proinflammatory cytokines..$^{3-8}$ Our results support the hypothesis that, in the early stage of severe acute pancreatitis, activation of various inflammatory cells, which release proinflammatory cytokines and anti-inflammatory cytokines, plays an important role in the pathogenesis of the disease. ${ }^{1}{ }^{2}$ In experimental pancreatitis, systemic release of IL-10 correlates with the course of acute pancreatitis and parallels the release of TNF. ${ }^{33}$ Endogenous IL-10 controls TNF- $\alpha$ production and plays a protective role in the local and systemic consequences of the disease. ${ }^{33}$

IL-11 has recently been reported to be a potent anti-inflammatory cytokine. ${ }^{21}$ We found that serum IL-11 levels on days two to four were significantly higher in patients with severe pancreatitis than in those with mild attacks, but this increase was not as great as that of IL-10. The acute phase proteins play an important role in body homoeostasis under different inflammatory conditions. IL- 6 is known to be the major inducer of the acute phase protein response and correlates closely with serum CRP. ${ }^{84}$ IL- 11 has been shown to stimulate the synthesis of acute phase proteins by hepatoma cells. ${ }^{20}$ However, IL-11 induced only minimal stimulation of acute phase protein production by human primary hepatocytes compared with IL- $6 .{ }^{35}$ In our series, serum IL-11 showed a weak correlation with serum CRP on days one and two. Gabay et $a l^{35}$ showed that circulating levels of IL-11 did not contribute significantly to the production of acute phase proteins in patients with rheumatoid arthritis and spondylarthropathy.

Early prediction of the severity of acute pancreatitis is important for adequate treatment. We previously reported that IL- 6 was the most useful of the various proinflammatory cytokines for early prediction of the prognosis of acute pancreatitis. ${ }^{8}$ This study shows that IL-10 is also a useful variable for early assessment of the prognosis of acute pancreatitis. The prognostic capacity of IL-10 appeared to be greater than that of IL-11 in our series (accuracy rate $84 \% v 64 \%$ on day one and $82 \%$ $v 74 \%$ on day two). Although assessment of CRP concentration is a simple and reliable test for determining the severity of acute pancreatitis, measurement of IL-10 was shown to have better sensitivity, specificity, and accuracy for staging the severity of acute pancreatitis on day one. IL-10 seems not to be any better than but is as good as CRP for staging the severity on day two (accuracy rate $82 \%$ v 84\%). IL-10 and IL-6 have been shown to be useful in monitoring patients after endoscopic retrograde cholangiopancreatography. ${ }^{29}$

In previous reports, patients with a fatal outcome showed the most markedly elevated concentrations of proinflammatory cytokines on days one and two. ${ }^{348}$ The serum levels of IL-10 in our mortality cases were also very high compared with other patients with severe pancreatitis (3.6 times the mean values in severe pancreatitis). Because the death rate from acute pancreatitis is about $5-10 \%$, a prospective study with a larger number of patients and a longer period of observation is needed to confirm the definite usefulness of serum IL-10 for predicting mortality.

In conclusion, serum IL-10 and IL-11 concentrations reflect the severity of acute pancreatitis. IL-10 is a useful variable for early prediction of the prognosis of acute pancreatitis.

This study was supported by a grant from the Veterans General Hospital-Taipei, Republic of China.

1 Rinderknecht $\mathrm{H}$. Fatal pancreatitis, a consequence of excessive leukocyte stimulation? Int F Pancreatol 1988;3:105-12.

Gross V, Leser HG, Heinisch A, et al. Inflammatory mediators and cytokines: new aspects of the pathophysiology and assessment of severity of acute pancreatitis? Hepatoassessment of severity of acut
Gastroenterol 1993;40:522-30.

3 Leser HG, Gross V, Scheibenbogen C, et al. Elevation of serum interleukin- 6 concentration precedes acute phase response and reflects severity in acute pancreatitis. Gastroenterology 1991;101:782-5

4 Viedman JA, Pérez-Mateo M, Dominguez JE, et al. Role of interleukin-6 in acute pancreatitis. Comparison with C-reactive protein and phospholipase A. Gut 1992;33: 1264-7.

5 Heath DI, Cruickshank A, Gudgeon M, et al. Role of interleukin- 6 in mediating the acute phase protein response and potential as an early means of severity assessment in acute pancreatitis. Gut 1993;34:41-5

6 Gross V, Leser HG, Andreesen R, et al. Interleukin-8 (IL-8) and neutrophil activation in acute pancreatitis. Eur $\mathcal{F}$ Clin Invest 1992;22:200-3.

7 Exley AR, Leese T, Holloday MP, et al. Endotoxaemia and serum tumor necrosis factor as prognostic markers in serum tumor necrosis factor as prognostic

8 Chen CC, Wang SS, Lee FY, et al. Proinflammatory cytokines in early assessment of the prognosis of acute pancreatitis. Am f Gastroenterol 1999;94:213-18.

9 Fiorentino DF, Zlotnik A, Mosmann TR, et al. IL-10 inhibits cytokine production by activated macrophages. $\mathcal{F}$ Immunol 1991;147:3815-22.

10 Raphe P, Nakoinz I, Sampson-Johannes A, et al. T lymphocyte inhibitor of human blood cell production of IL-1 and tumor necrosis factor. F Immunol 1992;148:808-14.

11 Willems F, Marchant A, Delville JP, et al. Interleukin-10 inhibits B7 and intercellular adhesion molecule-1 expression on human monocytes. Eur f Immunol 1994;24:1007-9.

12 Taga $\mathrm{K}$, Tosato G. IL-10 inhibits T cell proliferation and IL-2 production. f Immunol 1992;148:1143-8.

13 Marchant A, Devière J, Byl B, et al. Interleukin-10 production during septicaemia. Lancet 1994;343:707-8.

14 Rongione AJ, Kusske AM, Kwan K, et al. Interleukin 10 Rongione AJ, Kusske AM, Kwan K, et al. Interleukin 10
reduces the severity of acute pancreatitis in rats. Gastroenreduces the severity of acute
terology 1997;112:960-7.

15 Van Laethem JL, Marchant A, Delvaux A, et al. Interleukin 10 prevents necrosis in murine experimental acute pancreatitis. Gastroenterology 1995;108:1917-22.

16 Pezzilli R, Billi P, Miniero R, et al. Serum interleukin-10 in human acute pancreatitis. Dig Dis Sci 1997;42:1469-72

17 Wereszczynska-Siemiatkowska U, Dabrowski A, Gabryelewicz A, et al. Serum E-selectin (ES), interleukin-10 (IL-10), interleukin-6 (IL-6) and plasma polymorphonuclear elastase PMN-E in different severity forms of human acute pancreatitis (AP). Digestion 1998;59(suppl 3):484-5.

18 Neben S, Turner K. The biology of interleukin-11. Stem Cells 1993;11 (suppl 2):156-62.

19 Yin TG, Schendel P, Yang YC. Enhancement of in vitro and in vivo antigen-specific antibody responses by interleukin11. $\mathcal{E}$ Exp Med 1992;175:211-16.

20 Baumann H, Schendel P. Interleukin-11 regulates the hepatic expression of the same plasma protein genes as hepatic expression of the same plasma prote
interleukin-6. F Biol Chem 1991;266:20424-7.

21 Trepicchio WL, Bozza M, Pedneault G, et al. Recombinant human IL-11 attenuates the inflammatory response 
through down-regulation of proinflammatory cytokine through down-regulation of proinflammatory cytokine release an

22 Lavin M, McMahon MJ. APACHE II score for assessment and monitoring of acute pancreatitis. Lancet 1989;ii:201-5.

23 Wilson C, Heath DI, Imrie CW. Prediction of outcome in acute pancreatitis: a comparative study of APACHE II, clinical assessment and
7 Surg 1990;77:1260-4

24 Ranson JHC, Pasternak BS. Statistical methods for quantifying the severity of clinical acute pancreatitis. F Surg Res 1977;22:79-91.

25 Bradley EL III. A clinically based classification system for acute pancreatitis: summary of the International Symposium on Acute pancreatitis, Atlanta, GA, September 11 through 13, 1992. Arch Surg 1993;128:586-90.

26 Harmoinen A, Perko M, Gronroos P. Rapid quantitative determination of C-reactive protein using LKB 8600 reaction rate analyzer. Clin Chim Acta 1981;111:117-20.

27 Ransohoff DF, Feinstein AR. Problems of spectrum and bias in evaluating the efficacy of diagnostic tests. $N$ Engl F bias in evaluating the effic

28 Hanley JA, McNeil BJ. The meaning and use of the area under a receiver operating characteristic (ROC) curve. Radiology 1982;143:29-36.
29 Oezcueruemez-Porsch M, Kunz D, Hardt PD, et al. Diagnostic relevance on interleukin pattern, acute-phase proteins, and procalcitonin in early phase of post-ERCP pancreatitis. Dig Dis Sci 1998;43:1763-9.

30 van Deuren M, van der Ven-Jongekrijg J, Bartelink AKM, et al. Correlation between proinflammatory cytokines and antiinflammatory mediators and the severity of disease in meningococcal infections. F Infect Dis 1995;172:433-9.

31 Bone RC, Balk RA, Cerra FB, et al. Definitions for sepsis and organ failure and guidelines for the use of innovative therapies in sepsis. Chest 1992;101:1644-55.

32 Molloy RG, Mannick JA, Rodrick ML. Cytokines, sepsis and immunomodulation. Br f Surg 1993;80:289-97.

33 Van Laethem JL, Eskinazi R, Louis H, et al. Multisystemic production of interleukin 10 limits the severity of acute pancreatitis in mice. Gut 1998;43:408-13.

34 Castell JV, Gomez-Lechon MJ, David M, et al. Interleukin-6 is the major regulator of acute phase protein synthesis in adult human hepatocytes. FEBS Lett 1989;242:237-9.

35 Gabay C, Singwe M, Genin B, et al. Circulating levels of IL-11 and leukaemia inhibitory factor (LIF) do not significantly participate in the production of acute-phase proteins by the liver. Clin Exp Immunol 1996;105:260-5. 